\title{
Topological Persistence of the Unit Eigenvectors of a Perturbed Fredholm Operator of Index Zero
}

\author{
Raffaele Chiappinelli, Massimo Furi and Maria Patrizia Pera
}

\begin{abstract}
Let $A, C: E \rightarrow F$ be two bounded linear operators between real Banach spaces, and denote by $S$ the unit sphere of $E$ (or, more generally, let $S=g^{-1}(1)$, where $g$ is any continuous norm in $E$ ). Assume that $\mu_{0}$ is an eigenvalue of the problem $A x=\mu C x$, that the operator $L=A-\mu_{0} C$ is Fredholm of index zero, and that $C$ satisfies the transversality condition $\operatorname{Img} L+C(\operatorname{Ker} L)=F$, which implies that the eigenvalue $\mu_{0}$ is isolated (and when $F=E$ and $C$ is the identity implies that the geometric and the algebraic multiplicities of $\mu_{0}$ coincide).

We prove the following result about the persistence of the unit eigenvectors: Given an arbitrary $C^{1}$ map $M: E \rightarrow F$, if the (geometric) multiplicity of $\mu_{0}$ is odd, then for any real $\varepsilon$ sufficiently small there exists $x_{\varepsilon} \in S$ and $\mu_{\varepsilon}$ near $\mu_{0}$ such that $A x_{\varepsilon}+\varepsilon M\left(x_{\varepsilon}\right)=\mu_{\varepsilon} C x_{\varepsilon}$.

This result extends a previous one by the authors in which $E$ is a real Hilbert space, $F=E, A$ is selfadjoint and $C$ is the identity. We provide an example showing that the assumption that the multiplicity of $\mu_{0}$ is odd cannot be removed.
\end{abstract}

Keywords. Fredholm operators, nonlinear spectral theory, eigenvalues, eigenvectors Mathematics Subject Classification (2010). Primary 47J10, secondary 47A75, $35 \mathrm{P} 30$

\section{Introduction}

Let $E$ and $F$ be two real Banach spaces, and $g: E \rightarrow \mathbb{R}$ any continuous norm on $E$, not necessarily equivalent to the Banach norm $\|\cdot\|$. Let

$$
S=\{x \in E: g(x)=1\}
$$

R. Chiappinelli: Dipartimento di Ingegneria dell'Informazione e Scienze Matematiche, Via Roma 56, I-53100 Siena, Italy; raffaele.chiappinelli@unisi.it

M. Furi, M. P. Pera: Dipartimento di Matematica e Informatica, Via S. Marta 3, I-50139 Florence, Italy; massimo.furi@unifi.it; mpatrizia.pera@unifi.it 
denote the unit $g$-sphere of $E$ and consider the system

$$
\left\{\begin{aligned}
A x+\varepsilon M(x) & =\mu C x, \\
x & \in S,
\end{aligned}\right.
$$

where $A: E \rightarrow F$ and $C: E \rightarrow F$ are bounded linear operators, $\varepsilon$ and $\mu$ are real parameters, and $M: E \rightarrow F$ is a $C^{1}$ map.

By a solution of (1) we mean a triple $(x, \varepsilon, \mu) \in S \times \mathbb{R} \times \mathbb{R}$ satisfying the equation $A x+\varepsilon M(x)=\mu C x$. Since, in this case, the $g$-norm of $x$ is one, we say that $x$ is a unit eigenvector of the perturbed operator $A+\varepsilon M$ corresponding to the eigenvalue $\mu$ (or, simply, that $x$ is a unit $\mu$-eigenvector of $A+\varepsilon M$ ).

We assume that $\mu_{0}$ is an eigenvalue of the problem $A x=\mu C x$ (i.e. the operator $L=A-\mu_{0} C$ has a nontrivial kernel $\operatorname{Ker} L$ ), that $L$ is Fredholm of index zero, and that $C$ satisfies the transversality condition

$$
\operatorname{Img} L+C(\operatorname{Ker} L)=F \text {. }
$$

We will show (see Remark 3.1) that, $L$ being Fredholm of index zero, this condition is equivalent to

$$
\operatorname{Img} L \oplus C(\operatorname{Ker} L)=F
$$

as well as to

$$
(u \in \operatorname{Ker} L) \wedge(C u \in \operatorname{Img} L) \Longrightarrow u=0 .
$$

As we shall see, the transversality condition (2) implies that the eigenvalue $\mu_{0}$ is isolated. Moreover, when $F=E$ and $C$ is the identity, it implies that the geometric and the algebraic multiplicities of $\mu_{0}$ coincide. In fact, in this case, $\operatorname{Img} L \oplus \operatorname{Ker} L=E$.

The set of the unit $\mu_{0}$-eigenvectors of the unperturbed problem (i.e. when $\varepsilon=0$ ) is the finite dimensional sphere $\operatorname{Ker} L \cap S$. Under the assumption that this sphere is even dimensional (which means that the kernel of $L$ is odd dimensional), we prove (see Theorem 3.9) that it contains at least one unit $\mu_{0^{-}}$ eigenvector $x_{0}$ with the property that:

There exists a sequence $\left\{\left(x_{n}, \varepsilon_{n}, \mu_{n}\right)\right\}$ in $S \times(\mathbb{R} \backslash\{0\}) \times \mathbb{R}$ which converges to $\left(x_{0}, 0, \mu_{0}\right)$ and such that $A x_{n}+\varepsilon_{n} M\left(x_{n}\right)=\mu_{n} C x_{n}, \forall n \in \mathbb{N}$.

When this happens we say that such an $x_{0}$ is a bifurcation point for problem (1). This terminology is justified by the fact that, identifying the sphere Ker $L \cap S$ of the unit $\mu_{0}$-eigenvectors of $A$ with the set $(\operatorname{Ker} L \cap S) \times\{0\} \times\left\{\mu_{0}\right\}$ of trivial solutions of system (1), any neighborhood of $x_{0}$ (regarded as $\left(x_{0}, 0, \mu_{0}\right)$ ) contains nontrivial solutions, i.e. solutions which are not of the type $\left(x, 0, \mu_{0}\right)$.

Moreover, we prove two persistence results: one regarding the eigenvalues (Theorem 3.6) and the other one regarding the eigenvectors (Theorem 3.7). Roughly speaking, these results, put together, imply that: 
If $\varepsilon$ is small, there exist $\mu_{\varepsilon}$ close to $\mu_{0}$ (persistence of the eigenvalues) and $x_{\varepsilon} \in S$ close to Ker $L$ (persistence of the eigenvectors) such that $A x_{\varepsilon}+\varepsilon M\left(x_{\varepsilon}\right)=\mu_{\varepsilon} C x_{\varepsilon}$.

Of course, without loss of generality, we may assume $\mu_{0}=0$, and this will be done from now on. In fact, system (1) can be equivalently written as

$$
\left\{\begin{aligned}
L x+\varepsilon M(x) & =\lambda C x, \\
g(x) & =1,
\end{aligned}\right.
$$

where $\lambda=\mu-\mu_{0}$ and, we recall, $L=A-\mu_{0} C$.

Our results about (3) - in fact, about a slightly more general form of it are stated and proved in Section 3. They extend analogous ones obtained in [8] in which $E$ is a real Hilbert space, $F=E, A$ is selfadjoint and $C$ is the identity. The topological tool utilized in the proofs of the results in [8], the Lefschetz Fixed Point Theorem, seems not to work in our more general situation. Therefore, we make use of a different instrument: a degree for Fredholm maps of index zero between Banach spaces (actually, Banach manifolds) that was constructed in [1]. Section 2 is devoted to recall and summarize the main points of this construction which is based, among others, on the idea of oriented Fredholm map of index zero and of oriented homotopy between such maps.

In Section 4 we provide examples in which our results apply, as well as examples showing that the assumption that the (geometric) multiplicity of $\mu_{0}$ is odd cannot be dropped.

Finally, we point out that the origin of the investigations about the topological persistence of eigenvalues and eigenvectors goes back to an article of the first author in which the multiplicity of $\mu_{0}$ is one (see [6]).

\section{Preliminaries}

Let $f: X \rightarrow Y$ be a continuous map between two metric spaces. We recall that $f$ is said to be compact if $f(X)$ is relatively compact, and completely continuous if it is compact on any bounded subset of $X$. If for any $p \in X$ there exists a neighborhood $U$ of $p$ such that the restriction $\left.f\right|_{U}$ is compact, then $f$ is called locally compact. The map $f$ is said to be proper if $f^{-1}(K)$ is compact for any compact subset $K$ of $Y$ and locally proper if for any $p \in X$ there exists a closed neighborhood $U$ of $p$ such that the restriction $\left.f\right|_{U}$ is proper. Recall that a proper map sends closed sets into closed sets.

A multivalued map $\phi: X \multimap Y$ between two metric spaces is said to be upper semicontinuous if it has compact (possibly empty) values and for any open subset $V$ of $Y$ the upper inverse image of $V$, i.e. the set $\phi^{-1}(V)=\{x \in X: \phi(x) \subseteq V\}$, is an open subset of $X$. Clearly, the composition of upper semicontinuous maps is upper semicontinuous.

The following remark will be used several times in the sequel. 
Remark 2.1. Let $K$ be a compact subset of $X \times Y$ and, for any $x \in X$, denote by $K_{x}=\{y \in Y:(x, y) \in K\}$ the slice of $K$ at $x$. Then, the multivalued map $x \in X \mapsto K_{x}$ (whose graph is $K$ ) is upper semicontinuous. To see this, let $V$ be any open subset of $Y$ and assume, by contradiction, that the set $U=\left\{x \in X: K_{x} \subseteq V\right\}$ is not open. Then, there exists a sequence $\left\{x_{n}\right\}$ in $X \backslash U$ which converges to some $x_{0} \in U$. For any $n \in \mathbb{N}$, choose $y_{n} \in K_{x_{n}} \cap(Y \backslash V)$. Because of the compactness of $K$, we may assume $\left(x_{n}, y_{n}\right) \rightarrow\left(x_{0}, y_{0}\right) \in K$. Thus, $y_{0}$ belongs to $K_{x_{0}}$ which is a subset of $V$, contradicting the fact that $y_{0}$ also belongs to the closed set $Y \backslash V$.

2.1. Linear Fredholm operators and Fredholm maps. Let $E$ and $F$ be real Banach spaces. We recall that a bounded linear operator $L: E \rightarrow F$ is called Fredholm (see, e.g., [11]) if both $\operatorname{Ker} L$ and Coker $L:=F / \operatorname{Img} L$ have finite dimension. The index of $L$ is the integer

$$
\text { ind } L=\operatorname{dim} \operatorname{Ker} L-\operatorname{dim} \text { Coker } L \text {. }
$$

For short, a Fredholm operator of index $n$ will be also called a $\Phi_{n}$-operator, or a $\Phi$-operator if its index is not specified.

It is known that the image of a $\Phi$-operator between Banach spaces is necessarily closed. One can verify that any linear operator from $\mathbb{R}^{k}$ to $\mathbb{R}^{s}$ is Fredholm of index $k-s$.

Here are some fundamental properties in the framework of $\Phi$-operators:

1. The set $\Phi_{n}(E, F)$ of Fredholm operators from $E$ to $F$ of a given index $n$ is open in the Banach space $L(E, F)$ of bounded linear operators; consequently, so is the set $\Phi(E, F)$ of Fredholm operators.

2. The composition of two Fredholm operators of indices $m$ and $n$ is Fredholm of index $m+n$.

3. Given $L, K \in L(E, F)$, with $L$ Fredholm and $K$ compact, then $L+K$ is Fredholm and ind $(L+K)=$ ind $L$.

A nonlinear map $f: \Omega \rightarrow F$ of class $C^{1}$ on an open subset of $E$ is called Fredholm at a given $x \in \Omega$ if the derivative $f^{\prime}(x): E \rightarrow F$ is a (linear) Fredholm operator. In this case the index of $f$ at $x$ is the index of $f^{\prime}(x)$. The map $f$ is said to be Fredholm [of index $n$ ] on a subset $X$ of $\Omega$ if it is Fredholm [of index $n$ ] at any $x \in X$. Notice that if $f$ is Fredholm on $X$, its index depends continuously on $x \in X$ (in fact, due to the above property (1), it is locally constant). Consequently, if $X$ is connected, the index of $f$ is constant in $X$.

Hereafter, a nonlinear Fredholm map of index zero will be also called a $\Phi_{0}$-map. Notice that a $\Phi_{0}$-operator $L: E \rightarrow F$ is also a $\Phi_{0}$-map, being differentiable at any $x \in E$ with $L^{\prime}(x)=L$.

According to a result of S. Smale (see [10]), a Fredholm map defined on an open subset of a Banach space is locally proper. 
We summarize the notions of orientation, introduced in [1,2], of a $\Phi_{0}$-operator and of a $\Phi_{0}$-map.

Given $L \in \Phi_{0}(E, F)$, a bounded linear operator $A: E \rightarrow F$ with finite dimensional image is called a corrector of $L$ if $L+A$ is invertible. Notice that the set of correctors of $L$ is nonempty. This is true, and of crucial importance in what follows, even when $L$ does not need to be corrected (i.e. when it is invertible). On the set $\mathcal{C}(L)$ of correctors of $L$ one has an equivalence relation as follows. Let $A, B \in \mathcal{C}(L)$ be given and consider the following automorphism of $E$ :

$$
T=(L+B)^{-1}(L+A)=I-(L+B)^{-1}(B-A),
$$

where $I$ denotes the identity in $E$. The operator $K=I-T=(L+B)^{-1}(B-A)$ has clearly finite dimensional image. Hence, given any finite dimensional subspace $E_{0}$ of $E$ containing $K(E)$, the restriction of $T$ to $E_{0}$ is an automorphism. Consequently, its determinant is well defined and nonzero (it is 1 when $E_{0}$ is the trivial subspace $\{0\}$ of $E$, and this occurs only in the case when $T$ is the identity). It is easy to check that this number does not depend on the choice of $E_{0}$. Thus, it makes sense to define the determinant of $T$ as the determinant of the restriction of $T$ to any finite dimensional subspace of $E$ containing the image of $K$. One says that $A$ is equivalent to $B$ or, more precisely, $A$ is $L$-equivalent to $B$, if

$$
\operatorname{det}\left((L+B)^{-1}(L+A)\right)>0 .
$$

As shown in [1], this is an equivalence relation on $\mathcal{C}(L)$ with two equivalence classes.

Definition 2.2 (Algebraic orientation of a $\Phi_{0}$-operator). Let $L$ be a linear Fredholm operator of index zero between two real Banach spaces. Each one of the two equivalence classes of $\mathcal{C}(L)$ is called an orientation of $L$, and $L$ is oriented when an orientation is chosen.

Given an oriented operator $L$, we call positive correctors of $L$ the elements of its orientation.

Definition 2.3 (Natural algebraic orientation of an isomorphism). An oriented isomorphism $L$ is said to be naturally oriented if the trivial (i.e. null) operator is a positive corrector, and this orientation is called the natural orientation of $L$.

Definition 2.4 (Sign of an oriented $\Phi_{0}$-operator). Let $L \in \Phi_{0}(E, F)$ be oriented. Its sign is the integer

$$
\operatorname{sign} L=\left\{\begin{aligned}
+1 & \text { if } L \text { is invertible and naturally oriented } \\
-1 & \text { if } L \text { is invertible and not naturally oriented } \\
0 & \text { if } L \text { is not invertible }
\end{aligned}\right.
$$


An orientation of a Fredholm operator of index zero induces an orientation to any sufficiently close operator. Precisely, consider a Fredholm operator of index zero $L$ and a corrector $A$ of $L$. Since the set of the isomorphisms from $E$ into $F$ is open in the space $L(E, F)$ of bounded linear operators, $A$ turns out to be a corrector of every $T$ in a suitable neighborhood $W$ of $L$ in $L(E, F)$. Therefore, if $L$ is oriented and $A$ is a positive corrector of $L$, any $T \in W$ can be oriented regarding $A$ as a positive corrector of $T$.

Definition 2.5 (Topological orientation of a $\Phi_{0}(E, F)$-valued map). Let $X$ be a topological space and $h: X \rightarrow \Phi_{0}(E, F)$ a continuous map. An orientation of $h$ is a continuous choice of an orientation $\alpha(x)$ of $h(x)$ for each $x \in X$, where "continuous" means that for any $x \in X$ there exists $A \in \alpha(x)$ which is a positive corrector of $h(u)$ for any $u$ in a neighborhood of $x$. The map $h$ is orientable when it admits an orientation and oriented when an orientation is chosen.

It is possible to prove (see [2, Proposition 3.4]) that two equivalent correctors $A$ and $B$ of a given $L \in \Phi_{0}(E, F)$ remain $T$-equivalent for any $T$ in a neighborhood of $L$. This implies that the notion of "continuous choice of an orientation" in Definition 2.5 is equivalent to the following one:

- For any $x \in X$ and any $A \in \alpha(x)$, there exists a neighborhood $U$ of $x$ such that $A \in \alpha(u)$ for all $u \in U$.

One can easily show that if $h: X \rightarrow \Phi_{0}(E, F)$ is orientable and $X$ is connected, then it admits exactly two orientations (one opposite to the other). Moreover, by means of the theory of covering spaces, it is not difficult to prove that if $X$ is simply connected and locally path connected, then $h$ is orientable (see, e.g., [2]).

Definition 2.6 (Topological orientation of a $\Phi_{0}$-map). Let $f: U \rightarrow F$ be a Fredholm map of index zero defined on an open subset of $E$. An orientation of $f$ is an orientation, in the sense of Definition 2.5, of the derivative

$$
f^{\prime}: U \rightarrow \Phi_{0}(E, F)
$$

Moreover, $f$ is said to be orientable or oriented, according to the homonymous property of $f^{\prime}$.

We point out that if $L: E \rightarrow F$ is a $\Phi_{0}$-operator, then it is orientable if regarded as a $\Phi_{0}$-map; that is, in the sense of Definition 2.6. In fact, at any $x \in E$, the derivative $L^{\prime}(x)$ coincides with $L$, which can be "constantly" oriented according to Definition 2.2. Unless otherwise stated, for such an operator the two notions of orientation, the algebraic and the topological, will be identified. The same convention is assumed even when one considers the restriction $\left.L\right|_{U}$ of $L$ to any open subset $U$ of $E$. 
Let $U$ be open in $E$ and let $H: U \times[0,1] \rightarrow F$ be a continuous map. We say that $H$ is a homotopy of Fredholm maps of index zero or, simply, a $\Phi_{0}$-homotopy if it is continuously differentiable with respect to the first variable and any partial map $H_{s}:=H(\cdot, s)$ is a $\Phi_{0}$-map.

Definition 2.7 (Topological orientation of a $\Phi_{0}$-homotopy). Let

$$
H: U \times[0,1] \rightarrow F
$$

be a $\Phi_{0}$-homotopy. An orientation of $H$ is an orientation of the partial derivative

$$
\partial_{1} H: U \times[0,1] \rightarrow \Phi_{0}(E, F), \quad(x, s) \mapsto\left(H_{s}\right)^{\prime}(x),
$$

in the sense of Definition 2.5. Moreover, $H$ is orientable or oriented, according to the homonymous property of $\partial_{1} H$.

The following result regards an important property of the notion of orientability. Roughly speaking, it is a sort of continuous transport of an orientation along a homotopy (see [2, Theorem 3.14]).

Theorem 2.8 (Orientation transport). Let $H: U \times[0,1] \rightarrow F$ be a $\Phi_{0}$-homotopy. Given any $s \in[0,1]$, assume that the partial map $H_{s}$ is oriented. Then there exists and is unique an orientation of $H$ which is compatible with that of $H_{s}$.

2.2. The degree for oriented Fredholm maps of index zero. Let us now sketch the construction of the degree given in [1] (see also [2,4]) and recall its main properties.

Definition 2.9 (Admissible triples). Let $f: \Omega \rightarrow F$ be a Fredholm map of index zero, $U$ an open subset of $\Omega$, and $y \in F$. The triple $(f, U, y)$ is said to be admissible for the degree if $f^{-1}(y) \cap U$ is compact and $f$ is oriented in $U$ (i.e. if it is oriented the restriction of $f$ to $U$ ).

Remark 2.10. Let $f: \Omega \rightarrow F$ be a Fredholm map of index zero and $y \in F$. Given an open set $U$ whose closure $\bar{U}$ is contained in $\Omega$, assume that $f$ is proper on $\bar{U}$ and oriented in $U$. Then $(f, U, y)$ is admissible whenever $y \notin f(\partial U)$.

The degree is an integer valued function, deg, defined on the family of all the admissible triples, that satisfies some important properties listed below. Roughly speaking, if $(f, U, y)$ is admissible, $\operatorname{deg}(f, U, y)$ is an algebraic count of the solutions in $U$ of the equation $f(x)=y$. In fact, when $y$ is a regular value, the degree of $(f, U, y)$ is preliminarily defined as

$$
\operatorname{deg}(f, U, y)=\sum_{x \in f^{-1}(y) \cap U} \operatorname{sign} f^{\prime}(x) .
$$


Notice that this sum makes sense since any $f^{\prime}(x)$ is an oriented isomorphism and the set $f^{-1}(y) \cap U$ is finite, being compact and discrete. Observe also that if $L \in L(E, F)$ is an oriented isomorphism, $U$ is any open subset of $E$ and $y$ any point of $F$, then the triple $(L, U, y)$ is admissible and $\operatorname{deg}(L, U, y)$ is either sign $L$ or zero, depending on whether $L^{-1}(y)$ belongs to $U$ or not.

To define the degree of an arbitrary admissible triple $(f, U, y)$, choose any open subset $V$ of $U$ containing $f^{-1}(y) \cap U$ and such that $f$ is proper on the closure $\bar{V}$ of $V$ ( $V$ exists since Fredholm maps are locally proper - see [10]) and put

$$
\operatorname{deg}(f, U, y):=\operatorname{deg}(f, V, z),
$$

where $z$ is any regular value for the restriction of $f$ to $V$, sufficiently close to $y$. As shown in [1], this definition is well posed.

The properties of this degree are analogous to the ones of the LeraySchauder degree. Here we mention the principal ones:

i) (Normalization) Let the identity $I$ of $E$ be naturally oriented. Then

$$
\operatorname{deg}(I, E, 0)=1
$$

ii) (Additivity) If $(f, U, y)$ is an admissible triple and $U_{1}, U_{2}$ are two disjoint open subsets of $U$ such that $f^{-1}(y) \cap U \subseteq U_{1} \cup U_{2}$, then

$$
\operatorname{deg}(f, U, y)=\operatorname{deg}\left(f, U_{1}, y\right)+\operatorname{deg}\left(f, U_{2}, y\right) .
$$

iii) (Existence) Let $(f, U, y)$ be admissible. If

$$
\operatorname{deg}(f, U, y) \neq 0
$$

then the equation $f(x)=y$ has a solution in $U$.

iv) (Excision) If $(f, U, y)$ is an admissible triple and $U_{1}$ is an open subset of $U$ containing $f^{-1}(y) \cap U$, then

$$
\operatorname{deg}\left(f, U_{1}, y\right)=\operatorname{deg}(f, U, y) .
$$

v) (Translation invariance) If $(f, U, y)$ is an admissible triple, then

$$
\operatorname{deg}(f, U, y)=\operatorname{deg}(f-y, U, 0)
$$

vi) (Homotopy invariance) Let $H: U \times[0,1] \rightarrow F$ be an oriented $\Phi_{0}$-homotopy and let $y \in F$. If the set $H^{-1}(y)$ is compact, then $\operatorname{deg}(H(\cdot, s), U, y)$ does not depend on $s \in[0,1]$.

Given a $\Phi_{0}$-homotopy $H: U \times[0,1] \rightarrow F$ and $y \in F$, we point out that if $H$ admits a continuous extension $\bar{H}$ to $\bar{U} \times[0,1]$ and this extension is proper, then $H^{-1}(y)$ is compact provided that $\bar{H}(x, s) \neq y$ for all $(x, s) \in \partial U \times[0,1]$. 


\section{Results}

Let $E$ and $F$ be two real Banach spaces, and denote by $g: E \rightarrow \mathbb{R}$ any continuous norm on $E$, not necessarily equivalent to the Banach norm $\|\cdot\|$. Consider the system

$$
\left\{\begin{aligned}
L x+N(\varepsilon, x) & =\lambda C x \\
g(x) & =1
\end{aligned}\right.
$$

where $L: E \rightarrow F$ and $C: E \rightarrow F$ are bounded linear operators, $\lambda$ is a real parameter, and $N: \mathbb{R} \times E \rightarrow F$ is continuous, continuously differentiable with respect to the second variable and such that $N(0, x)=0$ for all $x \in E$.

Notice that (4) is slightly more general than system (3) in which $N(\varepsilon, x)=\varepsilon M(x)$.

A solution of (4) is a triple $(x, \varepsilon, \lambda) \in E \times \mathbb{R} \times \mathbb{R}$ satisfying the equation $L x+N(\varepsilon, x)=\lambda C x$ and such that $g(x)=1$. Since, in this case, the $g$-norm of $x$ is one, we say that $x$ is a unit eigenvector of the perturbed operator $L+N(\varepsilon, \cdot)$ corresponding to the eigenvalue $\lambda$.

We assume that $L$ is Fredholm of index zero with nontrivial kernel and that

$$
\operatorname{Img} L \oplus C(\operatorname{Ker} L)=F \text {. }
$$

As a consequence, there exists $\sigma>0$ such that $L-\lambda C$ is invertible if $0<|\lambda| \leq \sigma$. In fact, according to the decompositions $E=E_{1} \oplus \operatorname{Ker} L$ and $F=\operatorname{Img} L \oplus C(\operatorname{Ker} L)$, write $L-\lambda C$ in a block-matrix form as

$$
L-\lambda C=\left(\begin{array}{cc}
L_{11}-\lambda C_{11} & 0 \\
-\lambda C_{21} & -\lambda C_{22}
\end{array}\right)
$$

where $L_{11}: E_{1} \rightarrow \operatorname{Img} L$ and $C_{22}: \operatorname{Ker} L \rightarrow C(\operatorname{Ker} L)$ are isomorphisms. Thus, so is $L_{11}-\lambda C_{11}$ if $\lambda$ is sufficiently small. Consequently, for $\lambda$ small and different from zero, given any $\left(y_{1}, y_{2}\right) \in \operatorname{Img} L \times C(\operatorname{Ker} L)$, the system

$$
\left\{\begin{array}{r}
L_{11} x_{1}-\lambda C_{11} x_{1}=y_{1} \\
-\lambda C_{21} x_{1}-\lambda C_{22} x_{2}=y_{2}
\end{array}\right.
$$

has one and only one solution $\left(x_{1}, x_{2}\right) \in E_{1} \times \operatorname{Ker} L$. This means that $\lambda=0$ is an isolated eigenvalue for the problem $L x=\lambda C x$.

The following remark will be useful in order to check that condition (5) holds true for some examples in Section 4.

Remark 3.1. Condition (5) implies $\operatorname{dim} C(\operatorname{Ker} L)=$ codim $\operatorname{Img} L$. Thus, taking into account that codim $\operatorname{Img} L=\operatorname{dim} \operatorname{Ker} L$, it is equivalent to

$$
\operatorname{Img} L+C(\operatorname{Ker} L)=F \text {. }
$$


Moreover, it is also equivalent to

$$
(u \in \operatorname{Ker} L) \wedge(C u \in \operatorname{Img} L) \Longrightarrow u=0 .
$$

In fact, if (8) holds, the restriction of $C$ to $\operatorname{Ker} L$ is clearly injective and, consequently, $C(\operatorname{Ker} L)$ has the same dimension as $\operatorname{Ker} L$, which equals the codimension of $\operatorname{Img} L$. Thus, (5) is verified, since (8) implies also $\operatorname{Img} L \cap$ $C(\operatorname{Ker} L)=\{0\}$. The converse implication is also true, since, if (5) holds, one has $\operatorname{dim} C(\operatorname{Ker} L)$ equals codim $\operatorname{Img} L$, which is the same as $\operatorname{dim} \operatorname{Ker} L$. Hence $C$ is one-to-one on $\operatorname{Ker} L$, and this implies (8) since $\operatorname{Img} L \cap C(\operatorname{Ker} L)=\{0\}$.

Clearly, a triple of the type $(x, 0,0) \in E \times \mathbb{R} \times \mathbb{R}$ is a solution of (4) if and only if $x$ belongs to the finite dimensional sphere $\operatorname{Ker} L \cap S$, where

$$
S=\{x \in E: g(x)=1\}
$$

denotes the unit $g$-sphere of $E$. These distinguished solutions (those with $(\varepsilon, \lambda)=(0,0))$ will be called trivial and, consequently, all the others will be said nontrivial. Due to this distinction, it makes sense to introduce the following definition.

Definition 3.2 (Bifurcation points). A unit eigenvector $x_{0} \in \operatorname{Ker} L \cap S$ of $L$ (corresponding to the eigenvalue $\lambda=0$ ) will be called a bifurcation point of system (4) if any neighborhood of $\left(x_{0}, 0,0\right)$, called bifurcation triple, contains nontrivial solutions of (4).

Since, as already observed, $L-\lambda C$ is invertible for $|\lambda|>0$ small, one gets that any nontrivial solution $(x, \varepsilon, \lambda)$ of (4) sufficiently close to a bifurcation triple $\left(x_{0}, 0,0\right)$ must have $\varepsilon \neq 0$.

The following result can be found in [5] in a more general version. We give here an independent proof.

Lemma 3.3 (On the sign-jump). Let $\sigma$ be such that $L-\lambda C$ is invertible for $0<|\lambda| \leq \sigma$. Then, given any one of the two orientations (in the sense of Definition 2.5) of the map

$$
h:[-\sigma, \sigma] \rightarrow \Phi_{0}(E, F), \quad h(\lambda)=L-\lambda C,
$$

$\operatorname{sign} h(\lambda)$ changes crossing $\lambda=0$ if and only if Ker $L$ is odd dimensional.

Proof. Since $[-\sigma, \sigma]$ is simply connected, the map $h$ admits exactly two orientations. The choice of any one of them does not affect the existence or the nonexistence of a sign-jump at $\lambda=0$.

As in (6), write $L-\lambda C$ as

$$
h(\lambda)=\left(\begin{array}{cc}
L_{11}-\lambda C_{11} & 0 \\
-\lambda C_{21} & -\lambda C_{22}
\end{array}\right)
$$


and observe that

$$
A=\left(\begin{array}{cc}
0 & 0 \\
0 & -2 \sigma C_{22}
\end{array}\right)
$$

is a corrector of $h(\lambda)$ for any $\lambda \in[-\sigma, \sigma]$. Thus, the map $h$ can, and here will be, oriented by choosing $A$ as a positive corrector of any $h(\lambda)$. Therefore, for $0<|\lambda| \leq \sigma, \operatorname{sign} h(\lambda)=1$ if $A$ is $h(\lambda)$-equivalent to the trivial corrector of $h(\lambda)$, which is the null operator, and sign $h(\lambda)=-1$ otherwise. Namely,

$$
\operatorname{sign} h(\lambda)=\operatorname{sign} \operatorname{det}\left(h(\lambda)^{-1}(h(\lambda)+A)\right) .
$$

For $0<|\lambda| \leq \sigma$ one has $h(\lambda)^{-1}(h(\lambda)+A)=I+h(\lambda)^{-1} A$, which, according to the decomposition $E=E_{1} \oplus \operatorname{Ker} L$, takes the block-matrix form

$$
\left(\begin{array}{cc}
I_{11} & 0 \\
D(\lambda) & \frac{\lambda+2 \sigma}{\lambda} I_{22}
\end{array}\right)
$$

where $I_{11}$ and $I_{22}$ are the identities of $E_{1}$ and Ker $L$ respectively, and

$$
D(\lambda)=-C_{22}^{-1} C_{21}\left(L-\lambda C_{11}\right)^{-1} .
$$

Now, recalling the definition of a finite dimensional linear perturbation of the identity (see Section 2.1) and letting $n$ denote the dimension of Ker $L$, we get

$$
\operatorname{sign} h(\lambda)=\operatorname{sign} \operatorname{det}\left(\frac{\lambda+2 \sigma}{\lambda} I_{22}\right)=\operatorname{sign}\left(\frac{\lambda+2 \sigma}{\lambda}\right)^{n},
$$

which implies the assertion.

Lemma 3.5 below is of crucial importance in the proof of our results on the persistence of eigenvalues and eigenvectors. We need first the following lemma about locally proper maps.

Lemma 3.4 (see [3]). Let $E$ and $F$ be real Banach spaces and $H: \Omega \rightarrow F$ a continuous map from an open subset of $E \times \mathbb{R}^{k}$ into $F$. Assume that $H$ is continuously differentiable with respect to the first variable and such that $\partial_{1} H(x, s): E \rightarrow F$ is a Fredholm operator for all $(x, s) \in \Omega$. Then $H$ is locally proper.

Lemma 3.5. Let $L, C, N: E \rightarrow F$ be as in (4) and let $H: E \times \mathbb{R} \times \mathbb{R} \rightarrow F$ be the map given by

$$
H(x, \varepsilon, \lambda)=L x+N(\varepsilon, x)-\lambda C x .
$$

Then, the set $\Omega \subseteq E \times \mathbb{R} \times \mathbb{R}$ of all $(x, \varepsilon, \lambda)$ such that the first partial derivative $\partial_{1} H(x, \varepsilon, \lambda)$ of $H$ at $(x, \varepsilon, \lambda)$ is Fredholm of index zero is an open neighborhood of $E \times\{0\} \times\{0\}$. Moreover, $H$ is locally proper on $\Omega$. 
Proof. Observe first that

$$
\Omega=\left\{(x, \varepsilon, \lambda) \in E \times \mathbb{R} \times \mathbb{R}: \partial_{1} H(x, \varepsilon, \lambda) \in \Phi_{0}(E, F)\right\}
$$

is open in $E \times \mathbb{R} \times \mathbb{R}$, as the inverse image of the open subset $\Phi_{0}(E, F)$ of $L(E, F)$ under the continuous map $\partial_{1} H$. Moreover $\Omega$ contains $E \times\{0\} \times\{0\}$ since, at any $(x, 0,0), \partial_{1} H(x, 0,0)$ coincides with $L$, which is Fredholm of index zero by assumption. This proves our first assertion.

To prove the second one, according to Lemma 3.4, it is enough to show that the partial derivative $\partial_{1} H$ is a nonlinear Fredholm map on $\Omega$. This is true because of the definition of $\Omega$.

As above, let $S$ denote the unit $g$-sphere of $E$ and, given $c>0$, put

$$
S^{c}=\{x \in S: \operatorname{dist}(x, \operatorname{Ker} L)<c\},
$$

where $\operatorname{dist}(\cdot, \operatorname{Ker} L)$ denotes the distance function from the set $\operatorname{Ker} L$ (in the Banach norm $\|\cdot\|$ of $E$ ).

Theorem 3.6 (Persistence of the eigenvalues). Let $L, C, N: E \rightarrow F$ be as above. Assume that $\operatorname{Ker} L$ is odd dimensional. Then, given $c>0$, there exist $a>0$ and $b>0$ such that:

(1) the set

$$
\Gamma=\left\{(\varepsilon, \lambda) \in[-a, a] \times[-b, b]: L x+N(\varepsilon, x)=\lambda C x \text { for some } x \in S^{c}\right\}
$$

is compact;

(2) for any $\varepsilon \in[-a, a]$, the set of eigenvalues

$$
\Gamma_{\varepsilon}=\left\{\lambda \in[-b, b]: L x+N(\varepsilon, x)=\lambda C x \text { for some } x \in S^{c}\right\}
$$

is nonempty;

(3) the multivalued eigenvalue map $\varepsilon \in[-a, a] \longmapsto \Gamma_{\varepsilon}$ is upper semicontinuous;

(4) $\Gamma_{0}=\{0\}$.

Proof. Let $H: E \times \mathbb{R} \times \mathbb{R} \rightarrow F$ and $\Omega \subseteq E \times \mathbb{R} \times \mathbb{R}$ be as in (9) and (10) respectively. Recall that - by Lemma $3.5-\Omega$ contains $E \times\{0\} \times\{0\}$ and $H$ is locally proper on $\Omega$. let

Consider the unit $g$-ball $B=\{x \in E: g(x)<1\}$ of $E$ and, given any $c>0$,

$$
U^{c}=\{x \in B: \operatorname{dist}(x, \operatorname{Ker} L)<c\}
$$

be the "hamburger shaped" set of those points in $B$ whose distance from Ker $L$ (in the Banach norm) is less that $c$. Clearly $U^{c}$ is open in $E$ and its closure $\overline{U^{c}}$ contains the compact set $\operatorname{Ker} L \cap \bar{B}$. Since $H$ is locally proper on $\Omega$ and the 
subset $(\operatorname{Ker} L \cap \bar{B}) \times\{0\} \times\{0\}$ of $\Omega$ is compact, there exist positive constants $a, b$ and $c$ such that the closure

$$
\bar{W}=\overline{U^{c}} \times[-a, a] \times[-b, b]
$$

of the open subset

$$
W=U^{c} \times(-a, a) \times(-b, b)
$$

of $E \times \mathbb{R} \times \mathbb{R}$ is contained in $\Omega$ and $H$ is proper on $\bar{W}$. Consequently, the set

$$
K=H^{-1}(0) \cap \bar{W}
$$

is compact, as inverse image of a compact set under a proper map. Because of Remark 2.1, the multivalued map that associates to any $(\varepsilon, \lambda) \in[-a, a] \times[-b, b]$ the slice

$$
K_{(\varepsilon, \lambda)}=\{x \in E:(x, \varepsilon, \lambda) \in K\}=\left\{x \in \overline{U^{c}}: H(x, \varepsilon, \lambda)=0\right\}
$$

is upper semicontinuous. Thus, $U^{c}$ being open in $E$, the set

$$
V=\left\{(\varepsilon, \lambda) \in[-a, a] \times[-b, b]: K_{(\varepsilon, \lambda)} \subset U^{c}\right\}
$$

is open in the rectangle $R=[-a, a] \times[-b, b]$.

Since $H$ is proper on $\bar{W}=\overline{U^{c}} \times R$, so is on $\overline{U^{c}}$ any partial map $H(\cdot, \varepsilon, \lambda)$, with $(\varepsilon, \lambda) \in R$. Moreover, any such a map is Fredholm of index zero on its natural domain, which is the open subset

$$
\Omega_{(\varepsilon, \lambda)}=\{x \in E:(x, \varepsilon, \lambda) \in \Omega\}=\left\{x \in E: \partial_{1} H(x, \varepsilon, \lambda) \in \Phi_{0}(E, F)\right\}
$$

of $E$. Notice that any $\Omega_{(\varepsilon, \lambda)}$ contains $\overline{U^{c}}$ since $\bar{W} \subset \Omega$.

As already pointed out, assumption (5) implies that $L-\lambda C$ is invertible for $\lambda \neq 0$ sufficiently small. Hence, in case taking $b$ smaller, we may suppose that $H(\cdot, 0, \lambda)=L-\lambda C$ is invertible if $0<|\lambda| \leq b$. Thus, any pair $(0, \lambda)$, with $\lambda \in[-b, b], \lambda \neq 0$, belongs to $V$. In particular, $V$ contains the up and down middle points $(0, b)$ and $(0,-b)$.

Observe now that $\bar{W}$ is simply connected. Thus, in this set the map

$$
(x, \varepsilon, \lambda) \mapsto \partial_{1} H(x, \varepsilon, \lambda)
$$

is orientable with two possible orientations. Let us choose one of them. For example the one which makes the partial derivative

$$
\partial_{1} H(0, b, 0)=L-b C
$$

naturally oriented. Consequently, any partial map $H(\cdot, \varepsilon, \lambda)$, with $(\varepsilon, \lambda) \in R$, becomes an oriented Fredholm map of index zero on $U^{c}$. Since any such a map 
is proper on the closure $\overline{U^{c}}$ of $U^{c}$, according to Remark 2.10, $V$ coincides with the set of all $(\varepsilon, \lambda) \in R$ for which the triple $\left(H(\cdot, \varepsilon, \lambda), U^{c}, 0\right)$ is admissible for the degree. In fact, recalling that

$$
K_{(\varepsilon, \lambda)}=\left\{x \in \overline{U^{c}}: H(x, \varepsilon, \lambda)=0\right\},
$$

one has

$$
V=\left\{(\varepsilon, \lambda) \in R: H(x, \varepsilon, \lambda) \neq 0 \text { for all } x \in \partial U^{c}\right\} .
$$

As a consequence of the homotopy invariance property of the degree, the integer valued function

$$
(\varepsilon, \lambda) \in V \mapsto \operatorname{deg}\left(H(\cdot, \varepsilon, \lambda), U^{c}, 0\right)
$$

is locally constant. Moreover, because of Lemma 3.3 and the definition of degree, with the chosen orientation we have

$$
\operatorname{deg}\left(H(\cdot, 0, b), U^{c}, 0\right)=\operatorname{sign}(L-b C)=1
$$

and

$$
\operatorname{deg}\left(H(\cdot, 0,-b), U^{c}, 0\right)=\operatorname{sign}(L+b C)=-1 .
$$

Hence, the points $(0, b)$ and $(0,-b)$ lie in different connected components of $V$.

Now, let $\Gamma$ denote the complement of $V$ in $R$. That is, $\Gamma$ is the compact set of all $(\varepsilon, \lambda) \in R$ for which the triple $\left(H(\cdot, \varepsilon, \lambda), U^{c}, 0\right)$ is not admissible for the degree. Namely,

$$
\Gamma=\left\{(\varepsilon, \lambda) \in R: H(x, \varepsilon, \lambda)=0 \text { for some } x \in \partial U^{c}\right\} .
$$

We observe that a vector $x \in \partial U^{c}$ such that $H(x, \varepsilon, \lambda)=0$ for some $(\varepsilon, \lambda) \in R$, is not necessarily a unit eigenvector of our problem. That is, $(x, \varepsilon, \lambda)$ need not be a solution of system (4), since $g(x)$ could be less than 1 . In fact, in $\partial U^{c}$ there are points in the unit $g$-sphere $S$, but there are also points not (necessarily) in $S$ whose distance from Ker $L$ is $c$ (recall that $U^{c}$ is a " $2 c$-thick slice" of the unit $g$-ball $B=\{x \in E: g(x)<1\}$ containing Ker $L \cap B$ in the middle). However, taking $R$ smaller if necessary, we obtain that any such a vector $x \in \partial U^{c}$ is a unit eigenvector. That is, if $R$ is sufficiently small, thanks to the compactness of $K$ and the consequent upper semicontinuity of the multivalued $\operatorname{map}(\varepsilon, \lambda) \mapsto K_{(\varepsilon, \lambda)}$, one has

$$
\Gamma=\left\{(\varepsilon, \lambda) \in R: H(x, \varepsilon, \lambda)=0 \text { for some } x \in S^{c}\right\} .
$$

In fact, $K_{(0,0)}=\operatorname{Ker} L \cap \bar{B}$ is contained in the open subset

$$
O^{c}=\{x \in E: \operatorname{dist}(x, \operatorname{Ker} L)<c\}
$$


of $E$ and, consequently, $K_{(\varepsilon, \lambda)} \subset O^{c}$ for all $(\varepsilon, \lambda)$ in a neighborhood of $(0,0)$.

Finally, to get assertion (1) we need, if necessary, to reduce the width of $R$ a little bit: taking into account that $(0, \pm b) \in V$, we may assume that both the up and down horizontal sides of the rectangle $R$ are contained in $V$.

Now, as already observed, assertion (1) holds true being $\Gamma$ the complement of the relatively open subset $V$ of the rectangle $R$.

Assertion (2) follows from the fact that any vertical segment in $R$ meets $\Gamma$ since, like the points $(0, b)$ and $(0,-b)$, the two horizontal sides of $R$ lie in different components of $V=R \backslash \Gamma$.

Assertion (3), recalling Remark 2.1, is a consequence of the compactness of $\Gamma$. Assertion (4) is verified since $b$ is such that $L-\lambda C$ is invertible for $0<|\lambda| \leq b$.

Theorem 3.7 (Persistence of the eigenvectors). Let $L, C, N: E \rightarrow F$ be as above. Assume that $\operatorname{Ker} L$ is odd dimensional. Then, given $c>0$, there exist $a>0$ and $b>0$ such that:

(1) the set

$$
\Xi=\left\{(\varepsilon, x) \in[-a, a] \times S^{c}: L x+N(\varepsilon, x)=\lambda C x \text { for some } \lambda \in[-b, b]\right\}
$$

is compact;

(2) for any $\varepsilon \in[-a, a]$, the set of eigenvectors

$$
\Xi_{\varepsilon}=\left\{x \in S^{c}: L x+N(\varepsilon, x)=\lambda C x \text { for some } \lambda \in[-b, b]\right\}
$$

is nonempty;

(3) the multivalued eigenvector map $\varepsilon \in[-a, a] \vdash \Xi_{\varepsilon}$ is upper semicontinuous;

(4) $\Xi_{0}=\operatorname{Ker} L \cap S$.

Proof. Let the map $H: E \times \mathbb{R} \times \mathbb{R} \rightarrow F$, the rectangle $R=[-a, a] \times[-b, b]$, the "hamburger" $U^{c} \subseteq E$, and the compact set $K=H^{-1}(0) \cap\left(R \times \overline{U^{c}}\right)$ be as in the proof of Theorem 3.6. Consider the compact set $\Sigma=K \cap S$ and recall that (as shown in the final part of the proof of Theorem 3.6) $R$ is such that for any $(x, \varepsilon, \lambda) \in K$ one has $\operatorname{dist}(x, \operatorname{Ker} L)<c$. Therefore

$$
\Sigma=K \cap S^{c}=\left\{(x, \varepsilon, \lambda) \in[-a, a] \times[-b, b] \times S^{c}: L x+N(\varepsilon, x)=\lambda C x\right\} .
$$

That is, $\Sigma$ is the set of solutions $(x, \varepsilon, \lambda)$ of (4) which belong to $R \times O^{c}$, where $O^{c}=\{x \in E: \operatorname{dist}(x, \operatorname{Ker} L)<c\}$.

The set

$$
\Xi=\left\{(\varepsilon, x) \in[-a, a] \times S^{c}: L x+N(\varepsilon, x)=\lambda C x \text { for some } \lambda \in[-b, b]\right\}
$$

is compact being the projection of the compact set $\Sigma$ into $[-a, a] \times S^{c}$. This proves assertion (1). 
Assertion (2) follows from the analogous one in Theorem 3.6, since here the constants $a, b$ and $c$ are the same as in that result.

Assertion (3), recalling Remark 2.1, is a consequence of the compactness of $\Xi$. Assertion (4) is verified since $b$ is such that $L-\lambda C$ is invertible for $0<|\lambda| \leq b$.

Remark 3.8. With the notation of Theorems 3.6 and 3.7, in the case when $\Gamma_{\varepsilon}$ and $\Xi_{\varepsilon}$ are singletons for all $\varepsilon \in[-a, a]$, the multivalued maps $\varepsilon \mapsto \Gamma_{\varepsilon}$ and $\varepsilon \vdash \Xi_{\varepsilon}$ can be regarded as ordinary (single valued) maps. In this case, the upper semicontinuity coincides with the continuity.

Theorem 3.9 (Existence of bifurcation points). Let $L, C, N: E \rightarrow F$ be as above. Assume that $\operatorname{Ker} L$ is odd dimensional. Then, the finite dimensional unit $g$-sphere Ker $L \cap S$ contains at least one bifurcation point of problem (4).

Proof. Let the positive constants $a, b, c$ and the compact set $\Sigma$ be as in the proof of Theorem 3.7 .

Recall that any triple $(x, \varepsilon, \lambda) \in \Sigma$ is a solution of (4), and observe that the projection of $\Sigma$ into the rectangle $R=[-a, a] \times[-b, b]$ is just the set $\Gamma$ of Theorem 3.6. This set, because of the properties 1., 2. and 4. stated in this theorem, contains pairs $(\varepsilon, \lambda) \in \Gamma$ as close to $(0,0)$ as one wishes, but different from $(0,0)$.

Thus in $\Sigma$ there is a sequence $\left\{\left(x_{n}, \varepsilon_{n}, \lambda_{n}\right)\right\}$, with $\left(\varepsilon_{n}, \lambda_{n}\right) \neq(0,0)$ for all $n$, such that $\left(\varepsilon_{n}, \lambda_{n}\right) \rightarrow(0,0)$.

Because of the compactness of $\Sigma,\left\{x_{n}\right\}$ has at least one cluster point $x_{0}$, and this is a bifurcation point.

\section{Examples}

In this section we provide examples in which our results apply, as well as examples showing that the assumption that the (geometric) multiplicity of the eigenvalue $\lambda=0$ (for the unperturbed problem) is odd cannot be dropped.

The following is, if we may say so, a multi-example: it provides problems for which the multiplicity of the eigenvalue $\lambda=0$ is an arbitrary positive integer.

Example 4.1. Given $1 \leq p \leq \infty$ and $k \in \mathbb{N}$, let $L_{k}: \ell^{p} \rightarrow \ell^{p}$ be the bounded linear operator that associates to any $x=\left(\xi_{1}, \xi_{2}, \ldots\right) \in \ell^{p}$ the element

$$
L_{k} x=\left(0,0, \ldots, 0, \xi_{k+1}, \xi_{k+2}, \ldots\right) \text {, }
$$

and define $M: \ell^{p} \rightarrow \ell^{p}$ by

$$
M x=\left(-\xi_{2}, \xi_{1},-\xi_{4}, \xi_{3}, \ldots,-\xi_{2 i}, \xi_{2 i+1}, \ldots\right) .
$$


Observe that $L_{k}$ is Fredholm of index zero and its kernel is the $k$-dimensional space

$$
\text { Ker } L_{k}=\left\{x \in \ell^{p}: x=\left(\xi_{1}, \xi_{2}, \ldots \xi_{k}, 0,0, \ldots\right)\right\} .
$$

Hence, $\lambda_{0}=0$ is an eigenvalue of $L_{k}$ of geometric multiplicity $k$.

Consider the system

$$
\left\{\begin{aligned}
L_{k} x+\varepsilon M x & =\lambda x, \\
g(x) & =1,
\end{aligned}\right.
$$

where $g(x)$ is the $\ell^{\infty}$ norm $\|x\|_{\infty}$ on the space $\ell^{p}$, which is clearly continuous whatever is $p \in[1, \infty]$. Notice that the transversality condition $(7)$ is satisfied since, in this case, the operator $C$ is the identity. Incidentally we observe that this implies that the algebraic multiplicity of the eigenvalue $\lambda_{0}=0$ (of the unperturbed problem) is the same as the geometric one.

It is easy to verify that if $k$ is even then, for any $\varepsilon$ and for any $\lambda$ sufficiently small, the above equation has no solutions $x \neq 0$. On the other hand, if $k$ is odd, then according to Theorem 3.9 there exists a sequence $\left\{\left(x_{n}, \varepsilon_{n}, \lambda_{n}\right)\right\}$ which converges to a point $\left(x_{0}, 0,0\right)$ and such that

$$
\varepsilon_{n} \neq 0, \quad\left\|x_{n}\right\|_{\infty}=1, \quad L_{k} x_{n}+\varepsilon_{n} M x_{n}=\lambda_{n} x_{n}, \quad \forall n \in \mathbb{N} .
$$

For example, if $k=3$, then for any $\varepsilon \neq 0$ we get the eigenvalue

$$
\lambda_{\varepsilon}=\frac{1-\sqrt{1-4 \varepsilon^{2}}}{2}
$$

of $T_{3}+\varepsilon M$ to which corresponds the eigenspace spanned by the eigenvector

$$
v_{\varepsilon}=\left(0,0,1, \xi_{4}(\varepsilon), 0, \ldots\right),
$$

where $\xi_{4}(\varepsilon)=\frac{\sqrt{1-4 \varepsilon^{2}}-1}{2 \varepsilon}=-\varepsilon+\mathrm{o}(\varepsilon)$. Thus, no matter what $p \in[1, \infty]$ we have chosen, we get exactly two bifurcation points for the perturbed eigenvalue problem

$$
\left\{\begin{aligned}
L_{3} x+\varepsilon M x & =\lambda x, \\
\|x\|_{\infty} & =1,
\end{aligned}\right.
$$

namely $(0,0, \pm 1,0, \ldots)$.

The following is an apparently misleading example that could induce to turn over in one's mind that our results about the persistence of eigenvalues and unit eigenvectors are false.

Example 4.2. Consider the following second order differential equation depending on the real parameters $\varepsilon$ and $\lambda$ :

$$
x^{\prime \prime}+\varepsilon \cos t=\lambda x, \quad t \in \mathbb{R} .
$$


We are interested in $2 \pi$-periodic solutions $x$ of (11) normalized as follows:

$$
\sup _{t \in \mathbb{R}}|x(t)|=1 \text {. }
$$

To this purpose, we consider the problem

$$
\left\{\begin{aligned}
x^{\prime \prime}+\varepsilon \cos t & =\lambda x, \\
x(0) & =x(2 \pi), \\
x^{\prime}(0) & =x^{\prime}(2 \pi), \\
g(x) & =1,
\end{aligned}\right.
$$

where $g(x)=\sup \{|x(t)|: t \in[0,2 \pi]\}$. Clearly, all the solutions of (12) are $C^{\infty}$ and we will look for them in the Banach space

$$
C_{2 \pi}^{2}([0,2 \pi])=\left\{x \in C^{2}([0,2 \pi]): x(0)=x(2 \pi), x^{\prime}(0)=x^{\prime}(2 \pi)\right\} .
$$

In the notation of Section 3, set

$$
E=C_{2 \pi}^{2}([0,2 \pi]), \quad F=C([0,2 \pi])
$$

and define

$$
\begin{array}{rlrl}
L: E & \rightarrow F & \text { by } & L x=x^{\prime \prime}, \\
N: \mathbb{R} \times E \rightarrow F & \text { by } & N(\varepsilon, x)(t)=\varepsilon \cos t, \\
C: E \rightarrow F & \text { to be the inclusion. }
\end{array}
$$

As observed in the preliminaries, the operator $x \mapsto x^{\prime \prime}$, which is clearly Fredholm of index 2 between $C^{2}([0,2 \pi])$ and $F$, becomes of index 0 , when restricted to the 2-codimensional closed subspace $E$ of $C^{2}([0,2 \pi])$. The kernel of $L$ is the odd dimensional (actually 1-dimensional) subspace of $E$ of the constant functions, and

$$
\operatorname{Img} L=\left\{y \in F: \int_{0}^{2 \pi} y(t) d t=0\right\} .
$$

Thus, the transversality condition (7) is clearly satisfied and, as one can check directly, the operator $L-\lambda C: E \rightarrow F$ given by $x \mapsto x^{\prime \prime}-\lambda x$ is invertible for $\lambda \neq 0$ sufficiently small. Therefore, for such values of $\lambda$ the equation

$$
x^{\prime \prime}+\varepsilon \cos t=\lambda x
$$

has one and only one solution in $E$, and this is

$$
x_{(\varepsilon, \lambda)}(t)=\frac{\varepsilon}{1+\lambda} \cos t
$$

Thus, $g\left(x_{(\varepsilon, \lambda)}\right) \rightarrow 0$ as $(\varepsilon, \lambda) \rightarrow(0,0)$, and this is, apparently, in contrast with our persistence results (theorems 3.6 and 3.7), as well with the result about the existence of bifurcation points (Theorem 3.9).

We claim that there are no contradictions between this example and our results. And so? To highlight the pitfall we suggest the reader to check what happens for $\lambda=0$ and $\varepsilon$ arbitrary. 
The following is another example, with evident physical meaning, without persistence of unit eigenvectors. In this case the unperturbed problem has an eigenvalue $\mu_{0}=1$ whose multiplicity is 2 . The elementary details are left to the reader.

Example 4.3. Consider the problem

$$
\left\{\begin{aligned}
x^{\prime \prime}+2 \varepsilon x^{\prime}+\mu x & =0, \\
x(0) & =x(2 \pi), \\
x^{\prime}(0) & =x^{\prime}(2 \pi), \\
g(x) & =1,
\end{aligned}\right.
$$

where $g(x)=\sup \{|x(t)|: t \in[0,2 \pi]\}$, observe that $\mu_{0}=1$ is an eigenvalue when $\varepsilon=0$, and check that there are no solutions when $\varepsilon \neq 0$.

We close with one example of a system of two coupled second order differential equations in which condition (7) is satisfied and the eigenvalue of the unperturbed problem has multiplicity 3. Consequently, the persistence phenomenon occurs.

Example 4.4. Consider the following system of coupled differential equations with $2 \pi$-periodic boundary conditions:

$$
\left\{\begin{aligned}
x^{\prime \prime}+x+\varepsilon \cos t & =\lambda x \\
y^{\prime \prime}-x+\varepsilon x^{\prime \prime} & =\lambda y \\
x(0)=x(2 \pi), & x^{\prime}(0)=x^{\prime}(2 \pi) \\
y(0)=y(2 \pi), & y^{\prime}(0)=y^{\prime}(2 \pi)
\end{aligned}\right.
$$

The solutions $(x, y)$ of $(13)$ are all of class $C^{\infty}$ and we seek for them in the subspace $E$ of the Banach space $C^{2}\left([0,2 \pi], \mathbb{R}^{2}\right)$ of those pairs $(x, y)$ satisfying the $2 \pi$-periodic boundary conditions of system (13). Notice that $E$ has codimension 4 in $C^{2}\left([0,2 \pi], \mathbb{R}^{2}\right)$ and, consequently, the operator

$$
L: E \rightarrow F=C\left([0,2 \pi], \mathbb{R}^{2}\right) \quad \text { given by } \quad(x, y) \mapsto\left(x^{\prime \prime}+x, y^{\prime \prime}-x\right)
$$

is Fredholm of index zero. Elementary computations show that the unperturbed problem has $\lambda=0$ as an eigenvalue whose eigenspace $\operatorname{Ker} L$ is 3-dimensional and is spanned by the following pairs of functions:

$$
(\sin t,-\sin t), \quad(\cos t,-\cos t), \quad(0,1)
$$

Consider, in $E$, the continuous norm

$$
g((x, y))=\sup \{|x(t)|+|y(t)|: t \in[0,2 \pi]\}
$$


and let $S$ denote the unit $g$-sphere of $E$.

As we will see, the transversality condition (5) is satisfied with $C$ the inclusion of $E$ into $F$. Thus, according to Theorem 3.9, the 2-dimensional sphere Ker $L \cap S$ contains some bifurcation points. Let us see if we can find them.

When $\lambda=0$, the first equation $x^{\prime \prime}+x+\varepsilon \cos t=\lambda x$ has no $2 \pi$-periodic solutions, except in the case when $\varepsilon=0$, which is of no interest for our search for bifurcation points. Therefore we may suppose $\lambda \neq 0$ and, of course, small. In this case the first equation admits only one $2 \pi$-periodic solution:

$$
x_{(\varepsilon, \lambda)}(t)=\frac{\varepsilon}{\lambda} \cos t .
$$

Replacing this solution in the second differential equation we get

$$
y^{\prime \prime}-\lambda y=\frac{\varepsilon}{\lambda} \cos t+\frac{\varepsilon^{2}}{\lambda} \cos t,
$$

whose only $2 \pi$-periodic solution is

$$
y_{(\varepsilon, \lambda)}(t)=-\frac{\varepsilon(1+\varepsilon)}{\lambda(1+\lambda)} \cos t
$$

Since $g\left(\left(x_{(\varepsilon, \lambda)}, y_{(\varepsilon, \lambda)}\right)\right)=\left|\frac{\varepsilon}{\lambda}\right|\left(1+\left|\frac{1+\varepsilon}{1+\lambda}\right|\right)$, for $\varepsilon$ and $\lambda$ small, the solution $\left(x_{(\varepsilon, \lambda)}, y_{(\varepsilon, \lambda)}\right)$ of system (13) lies in the $g$-sphere $S$ if and only if

$$
1=\left|\frac{\varepsilon}{\lambda}\right|\left(2+\frac{\varepsilon-\lambda}{1+\lambda}\right) \cong 2\left|\frac{\varepsilon}{\lambda}\right|
$$

Consequently, the 2-dimensional sphere $\operatorname{Ker} L \cap S$ has exactly two (antipodal) bifurcation points:

$$
\frac{1}{2}( \pm \cos t, \mp \cos t)
$$

Finally, as promised, let us check that condition (5) is satisfied. To this purpose, we will show that the following equivalent condition (see (8) in Remark 3.1) holds true:

$$
(u \in \operatorname{Ker} L) \wedge(C u \in \operatorname{Img} L) \Longrightarrow u=0 .
$$

Let $u$ be a generic element in $\operatorname{Ker} L$. That is,

$$
u(t)=a(\sin t, \sin t)+b(\cos t, \cos t)+c(0,1), \quad a, b, c \in \mathbb{R} .
$$

We need to show that if the system

$$
\left\{\begin{array}{l}
x^{\prime \prime}+x=a \sin t+b \cos t \\
y^{\prime \prime}-x=a \sin t+b \cos t+c
\end{array}\right.
$$


has at least one $2 \pi$-periodic solution, then $a=b=c=0$.

The first equation has no $2 \pi$-periodic solutions, unless when $a=b=0$, and in this case has many of them, all of the type $x(t)=\alpha \sin t+\beta \cos t$. Replacing any one of these solutions in the second equation, we get

$$
y^{\prime \prime}=\alpha \sin t+\beta \cos t+c .
$$

But this equation has a $2 \pi$-periodic solution (if and) only if the average, in the interval $[0,2 \pi]$, of its second member is zero, which is true (if and) only if $c=0$.

\section{References}

[1] Benevieri, P. and Furi, M., A simple notion of orientability for Fredholm maps of index zero between Banach manifolds and degree theory. Ann. Sci. Math. Québec 22 (1998), 131 - 148.

[2] Benevieri, P. and Furi, M., On the concept of orientability for Fredholm maps between real Banach manifolds. Topol. Methods Nonlinear Anal. 16 (2000), $279-306$.

[3] Benevieri, P. and Furi, M., Bifurcation results for families of Fredholm maps of index zero between Banach spaces. Nonlinear Anal. Forum 6 (2001)(1), $35-47$.

[4] Benevieri, P. and Furi, M., A degree for locally compact perturbations of Fredholm maps in Banach spaces. Abstr. Appl. Anal. 2006, Art. ID 64764, 20 pp.

[5] Benevieri, P., Furi, M., Pera, M. P. and Spadini, M., About the sign of oriented Fredholm operators between Banach spaces. Z. Anal. Anwend. 22 (2003)(3), $619-645$.

[6] Chiappinelli, R., Isolated connected eigenvalues in nonlinear spectral theory. Nonlinear Funct. Anal. Appl. 8 (2003), 557 - 579.

[7] Chiappinelli, R., Furi, M. and Pera, M. P., Normalized eigenvectors of a perturbed linear operator via general bifurcation. Glasg. Math. J. 50 (2008), $303-318$.

[8] Chiappinelli, R., Furi, M. and Pera, M. P., Topological persistence of the normalized eigenvectors of a perturbed self-adjoint operator. Appl. Math. Lett. 23 (2010), 193 - 197.

[9] Chiappinelli, R., Furi, M. and Pera, M. P., A new theme in nonlinear analysis: continuation and bifurcation of the unit eigenvectors of a perturbed linear operator. Comm. Appl. Anal. 15 (2011), 299 - 312.

[10] Smale, S., An infinite dimensional version of Sard's theorem. Amer. J. Math. 87 (1965), $861-866$.

[11] Taylor, A. E. and Lay, D. C., Introduction to Functional Analysis. New York: John Wiley \& Sons 1980.

Received July 11, 2013; revised January 22, 2014 